\title{
Blockchain Technology in Business Organizations: A Scoping Review
}

\author{
Yang Li \\ HEC Montréal \\ yang.li@hec.ca \\ Alexis Perron-Brault \\ HEC Montréal \\ alexis.perron-brault@hec.ca
}

\author{
Thierry Marier-Bienvenue \\ École Polytechnique de Montréal \\ thierry.marier-bienvenue@polymtl.ca \\ Xinyi Wang \\ HEC Montréal \\ xinyi.wang@hec.ca
}

\author{
Guy Paré \\ HEC Montréal \\ guy.pare@hec.ca
}

\begin{abstract}
The scientific literature on blockchain technology is emerging but increasing rapidly. This review paper aims to provide a deeper understanding of the nature and scope of the extant literature on blockchain technology in the particular context of business organizations. To achieve our main objective, we searched five databases and screened 320 papers for inclusion. As a result of the search and screen process, we identified 39 relevant articles. Data coding was first pilot tested and then performed independently by two teams of researchers. All disagreements were reconciled by a third coder. Our findings reveal that most of the extant literature focuses on "how" blockchain technology works and, to a lesser extent, on the "what", i.e. its potential applications and usages in business organizations. For its part, the "why" question, which focuses on the organizational motivations for adopting blockchain technology, was scarcely discussed in prior literature. In short, our findings reveal that many issues and questions remain to be investigated. Based on a gap analysis, we propose a few promising avenues that shall guide future research efforts in this important topic.
\end{abstract}

\section{Introduction}

During the 1990s, the Internet emerged and changed the way to do business. It brought with it many innovations, such as electronic data interchange, online banking, online shopping, and electronic payment, to name but a few. More recently, Satoshi Nakamoto (an alias) published a white paper on a new online transactions system based on a distributed model called blockchain [1]. Blockchain is not an "in-our-face" innovation we can see and touch as a smartphone or a smart device. But when it comes to digital or web transactions (i.e. exchange of value, goods and services), blockchain is the answer to a question many of us have been asking since the dawn of the Internet age: How can we collectively trust what happens online? In his paper, Nakamoto [1] explains that his motivation for conceptualizing the blockchain technology was associated with the flaws in the intermediary trust model used by financial institutions.

One year after it was initially conceptualized by Nakamoto, blockchain technology was implemented as a core component of the digital currency bitcoin, where it serves as the public ledger for all transactions. Bitcoin has been the focus of this technology for several years, attracting many investors, entrepreneurs and banks, as well as criminals because of its increasing value and anonymity. From January 2016 to January 2017, the bitcoin blockchain grew from 50 gigabytes to 100 gigabytes in size [2]. Interestingly, in the recent WannaCry ransomware large-scale attack the hackers requested bitcoins as the sole mode of ransom payment.

But how does blockchain work concretely? We must think of it as a historical fabric underneath recording everything that happens exactly as it occurs. Then, the chain stitches that data into encrypted blocks that can never be modified and scatters the pieces across a worldwide network of distributed computers or "nodes." These nodes are called miners. They all share a copy of the public ledger. This ledger, called the blockchain, consists of blocks that contain several transactions which consist of modifications to accounts' balances. Once the 
network of nodes all agree on what is true (e.g., whether a specific account has enough funds to send a specific amount to another account), these transactions are compiled into a block using cryptography.

Figure 1 depicts the infrastructure for a transaction to be accepted and settled on the blockchain. Person A wants to send 5 bitcoins (BTC) to Person B. The transaction is then broadcasted to the network of miners that make sure the transaction is valid. Using cryptography and a sophisticated algorithm, the transaction is approved (or rejected) and compiled in the ledger, alongside many more transactions.

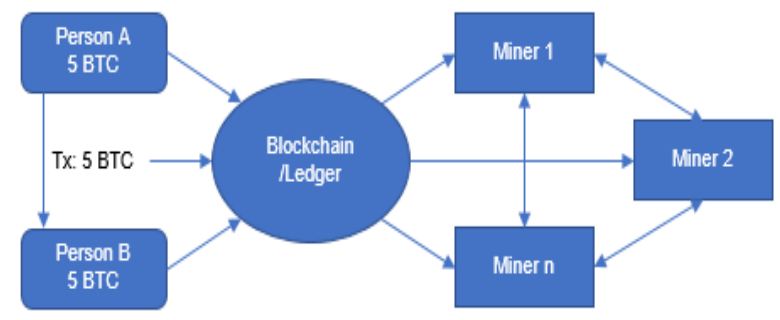

Figure 1. Blockchain transaction infrastructure

As the blockchain technology represents a breakthrough in the fields of cybersecurity, cryptography and peer-to-peer networks, most of the extant literature on this topic has been concerned with technical issues (e.g., [3]). However, blockchain is still considered an emerging topic in the business literature. In this regard, we posit that many nontechnical issues and questions must be addressed so that business executives and decision makers understand not only the intricacies of blockchain per se but also the types of business applications that are possible and how they could be used.

Because the non-technical literature on blockchain technology is rapidly expanding, we believe it is an appropriate time to scope prior knowledge on this topic, identify current gaps and suggest promising avenues for future research. The present review article aims to accomplish these objectives. More precisely, our main intent is to deepen our collective understanding of where the business literature on blockchain is at present and identify current gaps. Finally, we want to suggest a few promising research avenues for business scholars.

The remainder of this article is structured as follows. The next section outlines the methodology used to review the literature and the procedures used to ensure rigor and systematicity. The third section presents our main findings. Last, we discuss the results and propose a series of promising avenues for future work.

\section{Methodology}

To achieve our main goals, we followed Arksey and O'Malley's [4] and Levac et al.'s [5] guidelines on how to conduct a scoping review. The procedures proposed by these methodologists maximize both systematicity and transparency which, in turn, ensure a high level of rigor, reliability, and trustworthiness [6]. While scoping reviews are highly systematic in nature, they must not be confused with traditional systematic reviews. Indeed, whereas systematic reviews like meta-analyses attempt to integrate prior empirical findings on a mature topic in order to provide answers to questions like "what works" and "what works best," scoping reviews attempt to provide an initial indication of the size and nature of the available literature on an emerging topic, to identify gaps, and to propose a research agenda for future work [7]. Hence, the focus in scoping reviews is more on "what has been done" than on "what has been found." The main idea is to map the territory, which is why scoping reviews are also called mapping reviews. Next, we detail the different steps and activities that were performed.

\subsection{Developing a review protocol}

As an initial step, a formal and detailed review protocol was developed and followed throughout the entire review process. This protocol included the identification of the questions to be solved, the search strategy, the screening criteria and process, the data extraction strategy and procedures, the team members' responsibilities, the conceptual framework, the data analysis techniques, and the work schedule. As suggested by leading methodologists, the protocol was not conceived as a rigid tool which had to be applied in a strict manner. On the contrary, it served as a guiding framework which was modified as we saw fit. The broad questions included in the protocol are as follows: 1) what issues and questions have been investigated in the business literature on blockchain? 2) what are the main gaps in this literature? 3) what are examples of promising research avenues on blockchain for business researchers.

\subsection{Searching the literature}

To ensure that all types of papers were included in our sample and that our search was 
comprehensive, five databases were searched: ABI/INFORM Collection (ProQuest), Academic Search Complete (EBSCO), Emerald Insight, ScienceDirect and Web of Science. Because these databases include a rich, yet complementary collection of publishers and journals, searching through them allowed a comprehensive coverage and minimized the risk of selection bias.

To determine our set of keywords, each team member independently carried out a pilot test using the same database. After several rounds of tests, discussions and comparisons, the final keywords were as follows: "blockchain", "distributed ledger technology", "public ledger", and "computational trust". To maximize the breadth of coverage, we applied no time restriction on the search. However, only papers written in English were included in our sample.

Two team members searched through the first two databases while another duo searched through the remaining three databases. Every database was independently searched using the same keywords and search criteria. Then, members of each team compared their results to make sure that the search returned similar results. The search was conducted on April 2, 2017. It yielded a total of 320 papers $\left(\mathrm{n}_{\mathrm{ABI} / \mathrm{INFORM} \text { Collection [Proquest] }}=12, \mathrm{n}_{\text {Academic Search Complete }}\right.$ $[\mathrm{EBSCO}]=30, \mathrm{n}_{\text {Emerald Insight }}=12, \mathrm{n}_{\text {ScienceDirect }}=19$, web of Science $=247$ ).

\subsection{Screening papers}

To ensure that we consistently screened and selected the relevant papers for our study, five out of the 320 papers were randomly chosen for training purposes. All team members sat together and applied the inclusion and exclusion criteria in order to develop a shared understanding.

To be considered for further analysis, papers had to provide answers to at least one of the abovementioned research questions. Papers which strictly focused on bitcoin as well as those which solely investigated technical aspects of blockchain were excluded from our sample.

All 320 papers were screened by two sub-teams. The two members of each team independently screened the papers for which they were responsible. Then they compared and verified their results. The papers over which they disagreed were forwarded to a third researcher who made the final decision. The crosscheck process maximized the validity of the screening process. As shown in Figure 2, a total of 28 duplicates were found and 254 papers were excluded based on the abovementioned criteria. One additional paper was found based on a manual search leaving us with a final sample of 39 papers. The list of included papers is shown in Appendix A.

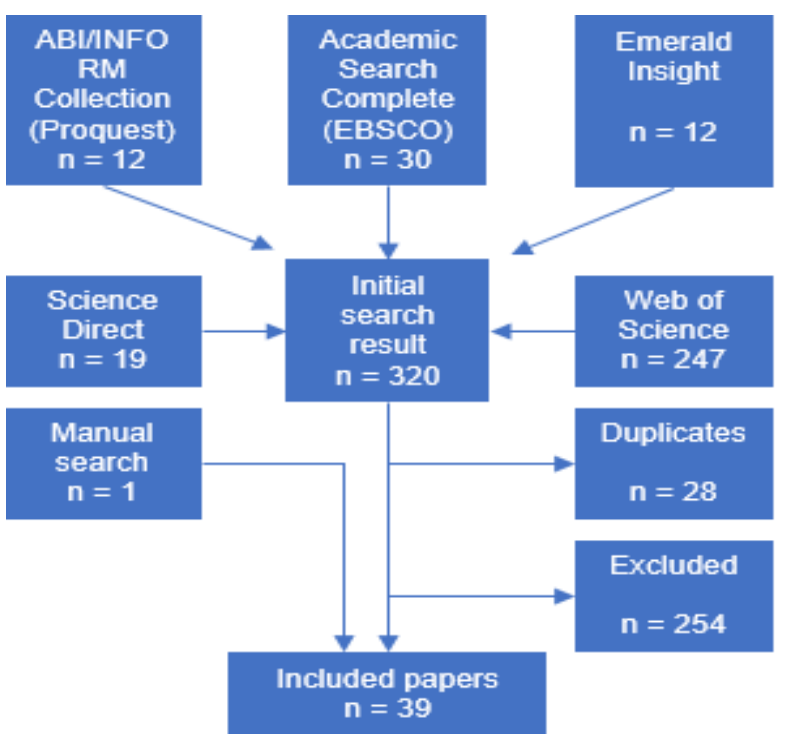

Figure 2. Flow diagram

\subsection{Charting the data}

During this step, a coding sheet was created for extracting data from the 39 papers. The coding form contains basic and core information about each paper. Basic information includes: year of publication, name of publication, paper title, paper type, and authors' background. In the core information section, research questions, research objectives, conclusions and ideas for future research were gathered. Again, our main goal was to clarify "what has been investigated" until now about blockchain in the business disciplines so that research gaps could be identified and a research agenda developed.

Our next task consisted of extracting the concepts at the heart of each paper, with the intention to develop a conceptual map of the blockchain applications and benefits. Porter and Millar's [8] value chain model appeared as an appropriate framework to perform this task. Indeed, the notion of value chain synthesizes all the primary activities which add value to a firm's products or services. It also includes inbound logistics, operations, outbound logistics, marketing, sales, service, procurement, human resources management, technological development and infrastructure. We thought this framework was comprehensive enough to help us map the blockchain applications in all types of organizations.

As a first step, we randomly selected six papers and sat together to extract data based on the value 
chain model. Our goal was to develop a shared understanding of the coding framework and coding process. However, the pilot exercise made us quickly realize that Porter and Millar's framework was not appropriate for characterizing potential blockchain applications and, hence, extracting data. Indeed, the topics discussed in the six papers were either very general (e.g., blockchain technology benefits in the financial sector [9]), or very specific (e.g., design of a blockchain application for managing personal medical data [10]). In addition, none of the surveyed studies discussed blockchain applications for marketing and sales, procurement, logistics or human resources, i.e. other key elements of the value chain model. It was therefore decided to put Porter and Millar's framework aside and try to find another classification scheme.

Reading through all of the six papers, we found that at least one of the following questions was addressed in all of them: What is blockchain? What potential usages can it have? How could blockchain be applied in certain industries or business contexts? Why should blockchain be applied? We also observed that different units of analysis were discussed. Indeed, some applications targeted specific individuals, such as patients, students or customers who want to have access to a wireless network; others targeted firms, either private or public (e.g., hospitals, universities) as well as governmental institutions.

Based on the pilot test, we decided to build our own classification scheme in order to achieve the abovementioned objectives. As shown in Table 1, the resulting scheme can be represented by a $3 \times 4$ matrix which refers to the level of blockchain application (individuals, firms or governments) and the focus of the study (what, why, whom or how). The "what" question refers to the nature of the blockchain applications; the "why" focuses on the incentives or reasons for investing in blockchain technology; the "whom" addresses the actors targeted by the blockchain technology; and the "how" question refers to the ways blockchain works and operates.

Coding of papers was divided equally between two teams of two researchers each. Papers were coded independently and all disagreements were reconciled by a third coder.

\subsection{Data analysis}

Two team members were responsible for jointly analyzing all the coding sheets. Alike most scoping reviews, descriptive statistics were computed to elucidate the nature and scope of the extent literature on the topic of interest [7]. Our key findings are presented in the following section.

\begin{tabular}{|c|c|c|c|c|}
\hline & \multicolumn{3}{|c|}{ Level of application } \\
\hline & & Individuals & $\begin{array}{c}\text { Firms (private } \\
\text { and public) }\end{array}$ & Governments \\
\hline \multirow{4}{*}{ 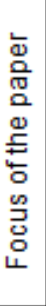 } & \begin{tabular}{|l} 
What \\
(usage)
\end{tabular} & & & \\
\hline & \begin{tabular}{|l} 
Why \\
(incentive)
\end{tabular} & & & \\
\hline & \begin{tabular}{|l}
$\begin{array}{l}\text { Whom } \\
\text { (people) }\end{array}$ \\
\end{tabular} & & & \\
\hline & $\begin{array}{l}\text { How } \\
\text { (process) }\end{array}$ & & & \\
\hline
\end{tabular}

Table 1. Classification scheme

\section{Results}

\subsection{Publication year and geographic distribution}

All included papers were published after 2014, a sign that interest in applications of blockchain in business organizations is quite recent. To be exact, seven papers $(17.9 \%)$ were published in 2015,29 in 2016 (74.4\%) and three (7.7\%) in early 2017. Based on a linear regression calculation, it was predicted that the number of papers to be published by the end of 2017 would be slightly above 40 .

For its part, Figure 3 indicates that prior research mainly comes from the United States, the United Kingdom and China. Altogether, those three countries produced more than half of all the studies included in our sample (56.4\%).

\subsection{Publication type and nature of studies}

In terms of publication type, Figure 4 shows that the vast majority of papers in our sample are conference proceedings $(64 \%)$, while about one-third are peer-reviewed journal papers (36\%). This can be interpreted as another sign that business research on this topic is still in its infancy. Only two pairs of papers come from the same publication source: the International Conference on Open and Big Data and the International Conference on Service-Oriented Computing. This also shows the diversity of the domains attempting to tackle this emerging topic.

Interestingly, our search revealed that it is only in September 2015 that the first peer-reviewed academic journal dedicated to blockchain technology research, called Ledger, was announced. Because the inaugural issue was published in December 2016 and the journal mainly covers aspects of mathematics, 
computer science and engineering, no article published in this new outlet was included in the present study.

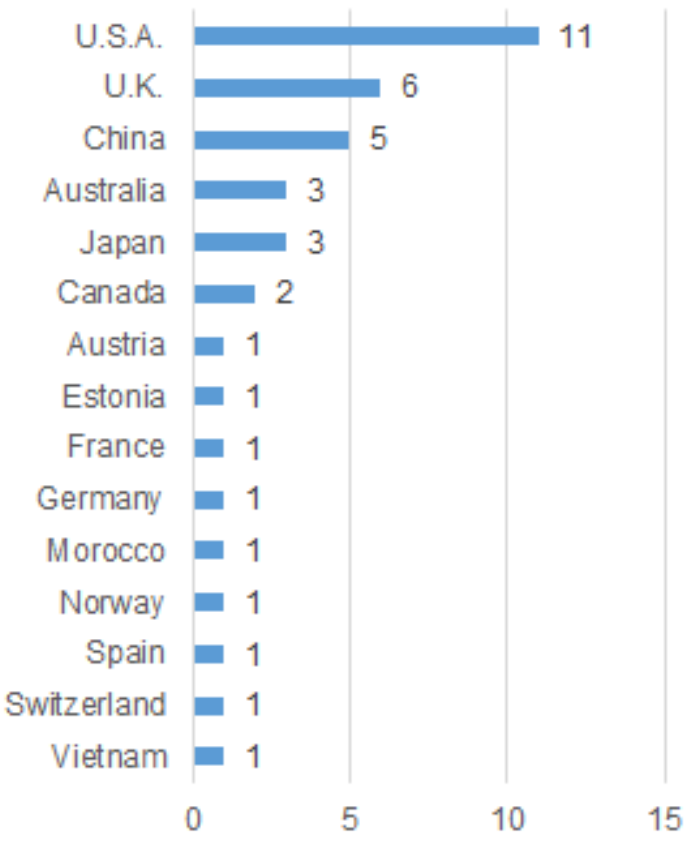

Figure 3. Origin of papers

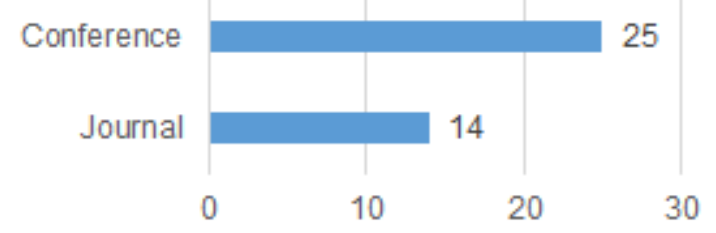

Figure 4. Type of publication

Figure 5 shows that most of the papers included in our sample are conceptual in nature $(79.5 \%)$, presenting ideas, concepts or theories about blockchain usages in the business world. Out of the 39 included papers, four $(10.3 \%)$ are qualitative empirical studies, one $(2.5 \%)$ is a quantitative study, while the remaining three $(7.7 \%)$ are opinion papers/editorials.

Figure 6 shows the number of papers per domain. Computer science, information systems/IT and software architecture represent $61.5 \%$ of the sample (24 papers). Finance also represents an important field of investigation (6 papers, 15.4\%) mainly because of the centrality of cryptocurrency in this domain. Other areas include law with three papers and sociology, pharmaceuticals and management with one paper each.

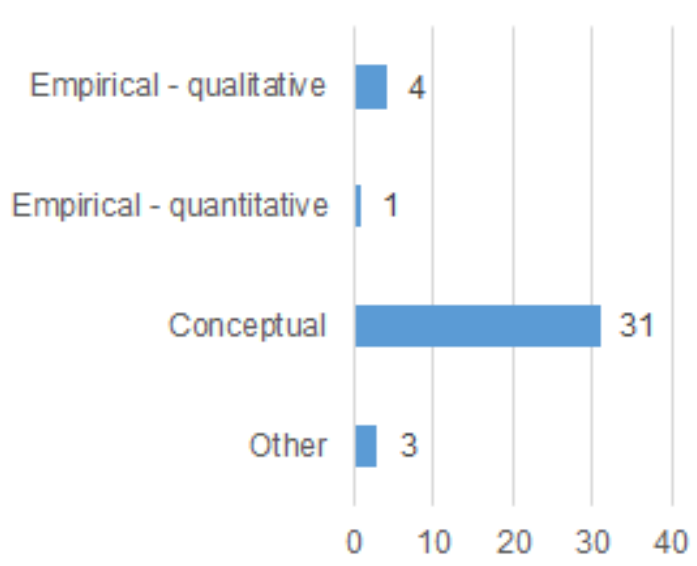

Figure 5. Nature of studies

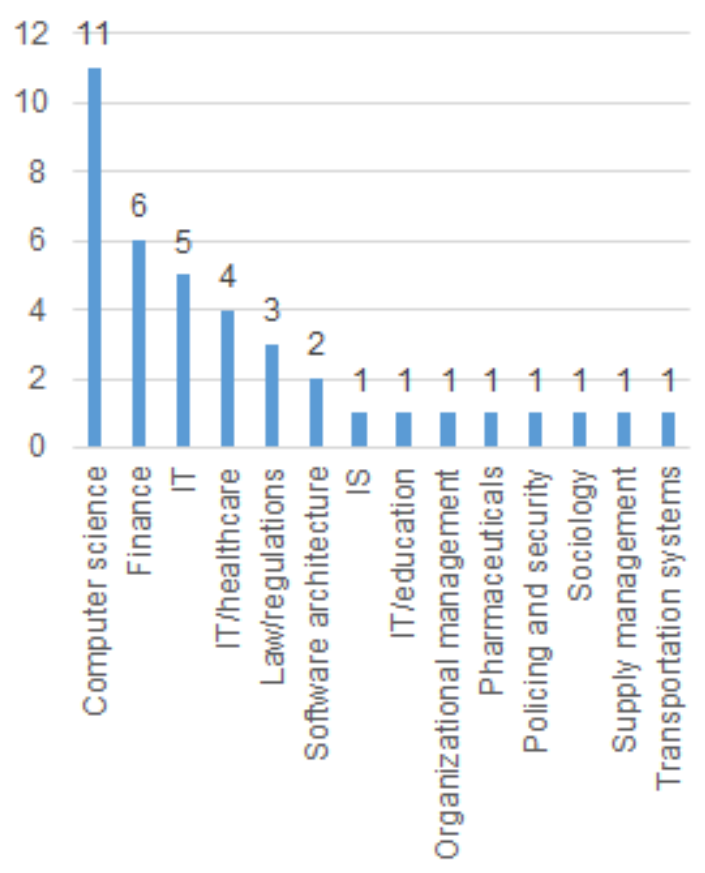

Figure 6. Diversity of domains $(n=39)$

\subsection{Main applications of blockchain in business organizations}

Most of the papers included in our sample (76.9\%) discuss various applications of blockchain in business organizations. The others concentrate on regulation issues (e.g., [11, 12]), advantages and disadvantages (e.g., $[13,15])$; user experience (e.g., [16]), impact (e.g., $[17,18])$; and opportunities, risks and challenges (e.g., [14]).

Among the 30 papers that elaborate on blockchain usages (see Figure 7), eight give general, yet narrative descriptions of possible applications. For instance, Mettler [19] shows how blockchain 
technology can be used to assist smart healthcare management, empower patient-generated health data and fight counterfeit drugs. For their part, Irwin and Milad [20] discuss how blockchain is being used as the base of bitcoin to fund violent jihads' acts of terror. Huckle et al. [21] provide examples of how blockchain can be used for autopay, foreign currency exchange and digital rights management. At a more macro level, Garrod [22] argues that blockchain will advance human development as the basis of a decentralized autonomous society based on blockchain technology.

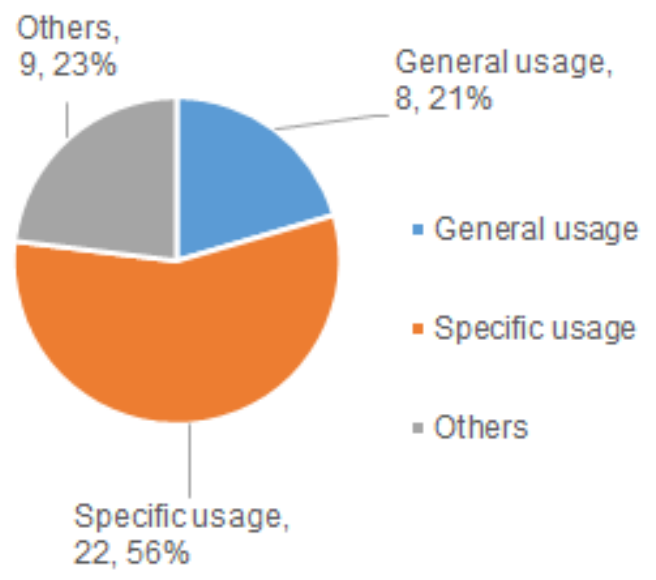

Figure 7. Forms of blockchain usages

Importantly, a total of 22 papers discuss specific applications of blockchain technology. Models are based either on mathematical formulas, flow charts or programming codes. The results are usually systems or platforms used for data storing, protecting, sharing and transforming. For instance, Lemieux [23] designs a system for creating and preserving trustworthy digital records. Kishigami et al. [24] and Fujimura et al. [25] both try to establish a digital rights management system for content distribution. For their part, Azaria et al. [26] and Yue et al. [10] developed solutions for patient data management; an electronic medical records management system and an application which is designed to deal with data control and data sharing under conditions of privacy protection. Dennis and Owen [27], Dennis and Owenson [28], Sharples and Domingue [29] and Yasin and Liu [30] present various systems modelling approaches for personal reputation management. The remaining papers mainly discuss the advantages of blockchain for building e-business models, designing intelligent transportation systems or getting access to Wi-Fi. The full list of specific blockchain usages is available upon request from the first author.

\subsection{Focus of inquiry and level of analysis}

As explained earlier, the included papers were classified according to a framework that we inductively developed. This framework includes the primary focus of inquiry (what, whom, how or why) and the level of analysis (individuals, firms or governments) of each paper. The results of this classification are shown in Table 2. It is important to note that since one paper could have more than one focus and more than one level, the total number is greater than 39. For that same reason, percentages also add up to more than $100 \%$.

\begin{tabular}{|c|c|c|c|c|}
\hline & \multicolumn{3}{|c|}{ Level of application } \\
\hline & & Individuals & $\begin{array}{c}\text { Firms (private } \\
\text { and public) }\end{array}$ & Governments \\
\hline \multirow{4}{*}{ 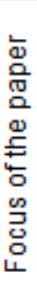 } & \begin{tabular}{|l} 
What \\
(usage)
\end{tabular} & 4 & 9 & 4 \\
\hline & $\begin{array}{l}\begin{array}{l}\text { Why } \\
\text { (incentive) }\end{array} \\
\end{array}$ & 1 & 2 & 1 \\
\hline & \begin{tabular}{|l} 
Whom \\
(people)
\end{tabular} & 2 & 2 & 0 \\
\hline & $\begin{array}{l}\text { How } \\
\text { (process) }\end{array}$ & 11 & 18 & 6 \\
\hline
\end{tabular}

Table 2. Focus of inquiry and level of application

Our results reveal that most of the papers $(61.5 \%)$ focus on the "how" question. Those papers often propose an explanation of the process behind a blockchain application in a specific business context. Examples are Sharples and Domingue's [29] proposition of a blockchain educational records system, Hull et al.'s [31] shared ledger business collaboration language and Gerstl's [32] use of blockchain to improve the uniform commercial code. Another important portion of the sample (35.9\%) consists of studies describing potential applications of the blockchain in the business context, without going into the details of how they work. These papers were therefore classified as mainly tackling the "what" question. Huckle et al.'s [21] effort on the coupling of blockchain with the Internet of things and Fanning and Centers' [18] discussion about the future impacts of blockchain on financial services are good examples of this. Interestingly, very few papers focus on the "whom" question (7.7\%). The "why" question, which emphasizes the incentives for adopting blockchain, is also dealt with in a minority of articles (5.1\%). Examples of these are Folkinshteyn and Lennon's [16] application of the Technology Acceptance Model (TAM) and Garrod's [22] analysis of the Decentralized Autonomous Organization (DAO). 
Finally, the level of application of the included papers is also unevenly distributed. Indeed, $74.4 \%$ of all papers in our sample focus on the firm level, studying possible applications to improve resource management or organizational efficiency. As shown in Table 2, applications at the individual and the government levels have been much less investigated as of today.

\section{Discussion}

The results of this scoping review reveal the current state of research on business applications of the blockchain technology. Our findings indicate that many issues and questions remain to be explored. As of today, most attention has been on ideation, i.e. possible applications and their related proofs-ofconcept. Most of those anticipated usages focus on specific online system designs for data storing, protecting, sharing and transforming; and the areas involved have concentrated on online data recording and finance. Examples are Azaria et al.'s [26] MedRec, an application for medical data access, and BRIGHT, a decentralized rights management system conceived by Fujimura et al. [25].

Hence, based on our analysis there are several gaps in the extent literature on blockchain. Due to space constraints, we discuss three of the most apparent gaps we identified. First, very few empirical studies have attempted to develop potential applications that go beyond record management systems and security issues. While those represent important areas with great opportunities, we think that there are other ideas to be explored in various domains. For example, the timestamps in blockchain can be used to serve time-sensitive tasks, such as just-in-time manufacturing (JIT). Indeed, JIT is a supply chain methodology aiming at reducing flow time and saving warehousing costs. It requires that suppliers send parts to the manufacturer at a specific time. On-time delivery is very important to keep the manufacturer's production running smoothly and efficiently. Early delivery of products may represent additional costs (e.g., extra warehouse renting cost) while late delivery can delay the entire production process. The timestamps in blockchain could then record the delivery time of parts, and the manufacturer may use those timestamps as triggers to start following manufacturing process at the appropriate time.

While the above illustration is only one example, we believe it will help us broaden and widen our perspectives so we better understand and appreciate the potentiality of blockchain technology. Indeed, over focusing on a few applications limits the potential of blockchain in businesses. We strongly encourage business scholars to investigate other types of usage like the one illustrated above. This will contribute to enriching our collective understanding and knowledge of blockchain technology. Such studies will also be of great value to practitioners in different industries who desire to take advantage of blockchain.

Second, there is also a lack of empirical studies examining the incentives leading business organizations to invest in and adopt blockchain technology. Indeed, knowledge about the reasons for adopting and using blockchain technology in private and public organizations is rather scarce. We suggest that future studies investigate the motivations associated with blockchain adoption and how these motivations influence how blockchain initiatives are implemented and managed in companies.

Last, but not least, the actual and anticipated impacts of blockchain on individuals, firms and governments (the "whom" question) are yet to be documented in the business literature. Indeed, blockchain's potential for business performance has not been investigated thoroughly. We posit that the perceived and actual impacts of blockchain on individuals, firms and governments merit scientific investigation at this stage of knowledge development. Formulating research problems about the impacts of blockchain technology is not only interesting, but also important and relevant. Novel explanations or theories might help us better understand in which context, under which circumstances and for whom blockchain technology works best.

Results of the present scoping review must be interpreted with caution due to some limitations. The first limitation is related to the search strategy, and more specifically to the language restriction. As mentioned earlier, we considered only papers written in English. While we believe most of the extent literature on blockchain has been published in English so far, during the search we actually found two papers written in other languages. The second limitation is related to the risk of selection bias. While the papers in our sample were retrieved from five databases that are commonly used in the social sciences disciplines, there is still a possibility that we missed some papers that might be relevant to our study. Due to pragmatic reasons, backward and forward searches have not yet been conducted but we anticipate to do so in the coming weeks. Third, and most importantly, we did not have the opportunity to validate our findings with a panel of experts, as suggested by Arksey and O'Malley [4]. We intend to do so in the coming months and share our results at 
the conference. Blockchain experts will likely provide valuable insights about relevant and important research avenues that the extant literature alone did not alert us to.

\section{Conclusion}

Our main objective in this review article was to determine the size and scope of the business literature on blockchain technology. Our findings reveal that most papers focused on how blockchain technology works in organizations and, to a much lesser extent, on the possible business applications of blockchain. However, prior studies barely investigated the incentives or motivations associated with this emerging technology, i.e. why blockchain technology should be adopted by private and public organizations, as well as the actual impacts blockchain provides to firms or organizations.

Based on these findings, we proposed some ideas for future research on this topic. In our viewpoint, future studies should focus on the "why" and the "whom" questions while also assessing the impacts of blockchain at the individual, firm and government levels. The reasons for applying the blockchain in organizations should be discussed more, so to demystify the possible impacts of this foundational technology. In this regard, we prevent business researchers from considering blockchain technology as a "black box" and future research should help practitioners better understand in which contexts and under which circumstances this technology works best, and for whom. Overall, we recommend that researchers tackle this important topic with a managerial mindset so that business executives and managers better understand what blockchain technology is all about, how it actually works, what types of benefits it can bring to various types of organizations, and in which circumstances it works best.

\section{References}

[1] Nakamoto, S. (2008). Bitcoin: A Peer-to-Peer Electronic Cash System.

[2] Blockchain Size. Blockchain Luxembourg. Available at: https://blockchain.info/charts/blocks-size?timespan=3years (last accessed on: 2017-08-21).

[3] Yli-Huumo, J., Ko, D., Choi, S., Park, S., \& Smolander, $\mathrm{K}$. (2016). Where is current research on Blockchain technology? - A systematic review. PLoS ONE, 11(10), 127.

[4] Arksey, H., \& O’Malley, L. (2005). Scoping studies: towards a methodological framework. International Journal of Social Research Methodology, 8(1), 19-32.
[5] Levac, D., Colquhoun, H., \& O'Brien, K. K. (2010). Scoping studies: advancing the methodology. Implementation Science, 5, article 69.

[6] Paré, G., Tate, M., Johnstone, D., \& Kitsiou, S. (2016). Contextualizing the twin concepts of systematicity and transparency in information systems literature reviews. European Journal of Information Systems, 25(6), 493-508.

[7] Paré, G., Trudel, M.C., Jaana, M. \& Kitsiou, S. (2015). Synthesizing Information Systems Knowledge: A Typology of Literature Reviews. Information \& Management, 52(2), 183-199.

[8] Porter, M. E., \& Millar, V. E. (1985). How information gives you competitive advantage. Harvard Business Review, 63(4), 149-152.

[9] Underwood, S. (2016). Blockchain beyond Bitcoin. Communications of the ACM, 59(11), 15-17.

[10] Yue, X., Wang, H. J., Jin, D. W., Li, M. Q., \& Jiang, W. (2016). Healthcare Data Gateways: Found Healthcare Intelligence on Blockchain with Novel Privacy Risk Control. Journal of Medical Systems, 40(10), 8.

[11] Kiviat, T. I. (2015). Beyond Bitcoin: Issues in Regulating Blockchain Transactions. Duke Law Journal, 65(3), 569-608.

[12] Yeoh, P. (2017). Regulatory issues in blockchain technology. Journal of Financial Regulation and Compliance, 25(2).

[13] Liu, P. T. S. (2016). Medical Record System Using Blockchain, Big Data and Tokenization. In K. Y. Lam, C. H. Chi, \& S. Qing (Eds.), Information and Communications Security, ICICS 2016 (Vol. 9977, pp. 254-261). Cham: Springer Int Publishing Ag.

[14] Nguyen, Q. K. (2016). Blockchain - A Financial Technology for Future Sustainable Development. 2016 3rd International Conference on Green Technology and Sustainable Development (Gtsd), 51-54.

[15] Tsai, W. T., Blower, R., Zhu, Y., \& Yu, L. (2016). A System View of Financial Blockchains. Proceedings 2016 IEEE Symposium on Service-Oriented System Engineering 2016, 450-457.

[16] Folkinshteyn, D., \& Lennon, M. (2016). Braving Bitcoin: A technology acceptance model (TAM) analysis. Journal of Information Technology Case and Application Research, 18(4), 220-249.

[17] Collomb, A., \& Sok, K. (2016). Blockchain / Distributed Ledger Technology (DLT): What Impact on the Financial Sector? Digiworld Economic Journal, 103(3), 93. [18] Fanning, K., \& Centers, D. P. (2016). Blockchain and Its Coming Impact on Financial Services. Journal of Corporate Accounting and Finance, 27(5), 53-57.

[19] Mettler, M. (2016). Blockchain Technology in Healthcare: The Revolution Starts Here. 2016 IEEE 18th International Conference on E-Health Networking, Applications and Services, 520-522.

[20] Irwin, A. S. M., \& Milad, G. (2016). The use of crypto-currencies in funding violent jihad. Journal of Money Laundering Control, 19(4), 407-425.

[21] Huckle, S., Bhattacharya, R., White, M., \& Beloff, N. (2016). Internet of Things, Blockchain and Shared Economy Applications. Procedia Computer Science, 58, 461-466. 
[22] Garrod, J. Z. (2016). The Real World of the Decentralized Autonomous Society. TriplecCommunication Capitalism \& Critique, 14(1), 62-77.

[23] Lemieux, V. L. (2016). Trusting records: is Blockchain technology the answer? Records Management Journal, 26(2), 110-139.

[24] Kishigami, J., Fujimura, S., Watanabe, H., Nakadaira, A., \& Akutsu, A. (2015). The Blockchain-based Digital Content Distribution System. Proceedings 2015 IEEE Fifth International Conference on Big Data and Cloud Computing, 187-190.

[25] Fujimura, S., Watanabe, H., Nakadaira, A., Yamada, T., Akutsu, A., Kishigami, J., \& Ieee. (2015). BRIGHT: A Concept for a Decentralized Rights Management System Based on Blockchain. 2015 IEEE 5th International Conference on Consumer Electronics, 345-346.

[26] Azaria, A., Ekblaw, A., Vieira, T., \& Lippman, A. (2016). MedRec: Using Blockchain for Medical Data Access and Permission Management. Proceedings 2016 2nd International Conference on Open and Big Data, 2530 .

[27] Dennis, R., \& Owen, G. (2015). Rep on the block : A next generation reputation system based on the blockchain. 10th International Conference for Internet Technology and Secured Transactions, 131-138.

[28] Dennis, R., \& Owenson, G. (2016). Rep on the Roll: A Peer to Peer Reputation System Based on a Rolling Blockchain. International Journal for Digital Society, 7(1), 1123-1134.

[29] Sharples, M., \& Domingue, J. (2016). The Blockchain and Kudos: A Distributed System for Educational Record, Reputation and Reward. In K. Verbert, M. Sharples, \& T. Klobucar (Eds.), Adaptive and Adaptable Learning, (vol. 9891, 490-496). Cham: Springer Int Publishing Ag.

[30] Yasin, A., \& Liu, L. (2016). An Online Identity \& Smart Contract Management System. In S. Reisman, S. I. Ahamed, L. Liu, D. Milojicic, W. Claycomb, M. Matskin, Z. Zhang (Eds.), IEEE 40th Annual Computer Software and Applications Conference Workshops (pp. 192-198). New York: IEEE.

[31] Hull, R., Batra, V. S., Chen, Y. M., Deutsch, A., Heath, F. F. T., \& Vianu, V. (2016). Towards a Shared Ledger Business Collaboration Language Based on DataAware Processes. In Q. Z. Sheng, E. Stroulia, S. Tata, \& S. Bhiri (Eds.), Service-Oriented Computing (vol. 9936, 1836). Cham: Springer Int Publishing Ag.

[32] Gerstl, D. S. (2016). Leveraging Bitcoin Blockchain Technology to Modernize Security Perfection under the Uniform Commercial Code. In A. Maglyas \& A. L. Lamprecht (Eds.), Software Business (vol. 240, 109-123). Cham: Springer Int Publishing Ag.

\section{Appendix A. List of included papers}

1. Apte, S., \& Petrovsky, N. (2016). Will blockchain technology revolutionize excipient supply chain management? Journal of Excipients and Food Chemicals, 7(3), 76-78.

2. Azaria, A., Ekblaw, A., Vieira, T., \& Lippman, A. (2016). MedRec: Using Blockchain for Medical Data
Access and Permission Management. Proceedings 2016 2nd International Conference on Open and Big Data - Obd 2016, 25-30.

3. Baur, A. W., Buhler, J., Bick, M., \& Bonorden, C. S. (2015). Cryptocurrencies as a Disruption? Empirical Findings on User Adoption and Future Potential of Bitcoin and Co. In M. Janssen, M. Mantymaki, J. Hidders, B. Klievink, W. Lamersdorf, B. VanLoenen, \& A. Zuiderwijk (Eds.), Open and Big Data Management and Innovation, I3e 2015 (Vol. 9373, pp. 63-80). Cham: Springer Int Publishing Ag.

4. Collomb, A., \& Sok, K. (2016). Blockchain / Distributed Ledger Technology (DLT): What Impact on the Financial Sector? Digiworld Economic Journal, 103(3), 93.

5. Dennis, R., \& Owen, G. (2015). Rep on the block: A next generation reputation system based on the blockchain. 2015 10th International Conference for Internet Technology and Secured Transactions (Icitst), 131-138.

6. Dennis, R., \& Owenson, G. (2016). Rep on the Roll: A Peer to Peer Reputation System Based on a Rolling Blockchain. International Journal for Digital Society, 7(1), 1123-1134.

7. Fanning, K., \& Centers, D. P. (2016). Blockchain and Its Coming Impact on Financial Services. Journal of Corporate Accounting and Finance, 27(5), 53-57.

8. Folkinshteyn, D., \& Lennon, M. (2016). Braving Bitcoin: A technology acceptance model (TAM) analysis. Journal of Information Technology Case and Application Research, 18(4), 220-249.

9. Fujimura, S., Watanabe, H., Nakadaira, A., Yamada, T., Akutsu, A., Kishigami, J., \& Ieee. (2015). BRIGHT: A Concept for a Decentralized Rights Management System Based on Blockchain. 2015 Ieee 5th International Conference on Consumer Electronics - Berlin (Icce-Berlin), 345-346.

10. Garrod, J. Z. (2016). The Real World of the Decentralized Autonomous Society. TriplecCommunication Capitalism \& Critique, 14(1), 62-77.

11. Gerstl, D. S. (2016). Leveraging Bitcoin Blockchain Technology to Modernize Security Perfection under the Uniform Commercial Code. In A. Maglyas \& A. L. Lamprecht (Eds.), Software Business (Vol. 240, pp. 109-123). Cham: Springer Int Publishing Ag.

12. Huckle, S., Bhattacharya, R., White, M., \& Beloff, N. (2016). Internet of Things, Blockchain and Shared Economy Applications. Procedia Computer Science, 58, 461-466.

13. Hull, R., Batra, V. S., Chen, Y. M., Deutsch, A., Heath, F. F. T., \& Vianu, V. (2016). Towards a Shared Ledger Business Collaboration Language Based on Data-Aware Processes. In Q. Z. Sheng, E. Stroulia, S. Tata, \& S. Bhiri (Eds.), Service-Oriented Computing (Vol. 9936, pp. 18-36). Cham: Springer Int Publishing Ag.

14. Irwin, A. S. M., \& Milad, G. (2016). The use of crypto-currencies in funding violent jihad. Journal of Money Laundering Control, 19(4), 407-425.

15. Jacynycz, V., Calvo, A., Hassan, S., \& Sanchez-Ruiz, A. A. (2016). Betfunding: A Distributed Bounty- 
Based Crowdfunding Platform over Ethereum. In S. Omatu, A. Selamat, G. Bocewicz, P. Sitek, I. Nielsen, J. A. GarciaGarcia, \& J. Bajo (Eds.), Distributed Computing and Artificial Intelligence (Vol. 474, pp. 403-411). Cham: Springer Int Publishing Ag.

16. Kianmajd, P., Rowe, J., \& Levitt, K. (2016). PrivacyPreserving Coordination for Smart Communities. 2016 IEEE Conference on Computer Communications Workshops, 1-2.

17. Kishigami, J., Fujimura, S., Watanabe, H., Nakadaira, A., \& Akutsu, A. (2015). The Blockchain-based Digital Content Distribution System. Proceedings 2015 Ieee Fifth International Conference on Big Data and Cloud Computing Bdcloud 2015, 187-190.

18. Kiviat, T. I. (2015). Beyond Bitcoin: Issues in Regulating Blockchain Transactions. Duke Law Journal, 65(3), 569-608.

19. Lemieux, V. L. (2016). Trusting records: is Blockchain technology the answer? Records Management Journal, 26(2)

20. Liu, P. T. S. (2016). Medical Record System Using Blockchain, Big Data and Tokenization. In K. Y. Lam, C. H. Chi, \& S. Qing (Eds.), Information and Communications Security, Icics 2016 (Vol. 9977, pp. 254-261). Cham: Springer Int Publishing Ag.

21. Mettler, M. (2016). Blockchain Technology in Healthcare The Revolution Starts Here. 2016 Ieee 18th International Conference on E-Health Networking, Applications and Services (Healthcom), 520-522.

22. Nguyen, Q. K. (2016). Blockchain - A Financial Technology for Future Sustainable Development. 2016 3rd International Conference on Green Technology and Sustainable Development (Gtsd), 5154

23. Norta, A. (2016). Establishing Distributed Governance Infrastructures for Enacting Cross-Organization Collaborations. In A. Norta, W. Gaaloul, G. R. Gangadharan, \& H. K. Dam (Eds.), Service-Oriented Computing - Icsoc 2015 Workshops (Vol. 9586, pp. 24-35). Cham: Springer Int Publishing Ag

24. Ouaddah, A., Abou Elkalam, A., \& Ouahman, A. A. (2017). Towards a Novel Privacy-Preserving Access Control Model Based on Blockchain Technology in IoT. In A. Rocha, M. Serrhini, \& C. Felgueiras (Eds.), Europe and Mena Cooperation Advances in Information and Communication Technologies (Vol. 520, pp. 523-533). Berlin: Springer-Verlag Berlin.

25. Roman, D., \& Stefano, G. (2016). Towards a Reference Architecture for Trusted Data Marketplaces similar to The Credit Scoring Perspective similar to. Proceedings 2016 2nd International Conference on Open and Big Data - Obd 2016, 95-101.

26. Sanda, T., \& Inaba, H. (2016). Proposal of New Authentication Method in Wi-Fi Access Using Bitcoin 2.0. 2016 Ieee 5th Global Conference on Consumer Electronics, 5.

27. Sharples, M., \& Domingue, J. (2016). The Blockchain and Kudos: A Distributed System for Educational Record, Reputation and Reward. In K. Verbert, M.
Sharples, \& T. Klobucar (Eds.), Adaptive and Adaptable Learning, Ec-Tel 2016 (Vol. 9891, pp. 490-496). Cham: Springer Int Publishing Ag

28. Swan, M. (2016). The Future of Brain-Computer Interfaces: Blockchaining Your Way into a Cloudmind, 60-82.

29. Tian, F. (2016). An Agri-food Supply Chain Traceability System for China Based on RFID \& Blockchain Technology. In B. J. Yang, J. Chen, X. Q. Cai, K. D. Qin, \& C. Zhou (Eds.), 2016 13th International Conference on Service Systems and Service Management. New York: Ieee.

30. Tsai, W. T., Blower, R., Zhu, Y., \& Yu, L. (2016). A System View of Financial Blockchains. Proceedings 2016 Ieee Symposium on ServiceOriented System Engineering Sose 2016, 450-457.

31. Underwood, S. (2016). Blockchain beyond Bitcoin. Communications of the Acm, 59(11), 15-17.

32. Xu, X. W., Pautasso, C., Zhu, L. M., Gramoli, V., Ponomarev, A., Tran, A. B. (2016). The Blockchain as a Software Connector. 2016 13th Working Ieee/Ifip Conference on Software Architecture (Wicsa), 182191.

33. Xu, X., Weber, I., Staples, M., Zhu, L., Bosch, J., Bass, L. Rimba, P. (2017). A Taxonomy of Blockchain-Based Systems for Architecture Design, (March)

34. Yasin, A., \& Liu, L. (2016). An Online Identity \& Smart Contract Management System. In S. Reisman, S. I. Ahamed, L. Liu, D. Milojicic, W. Claycomb, M. Matskin, Z. Zhang (Eds.), Proceedings 2016 Ieee 40th Annual Computer Software and Applications Conference Workshops (pp. 192-198). New York: Ieee.

35. Yeoh, P. (2017). Regulatory issues in blockchain technology. Journal of Financial Regulation and Compliance, 25(2)

36. Yuan, Y., \& Wang, F. Y. (2016). Towards Blockchain-based Intelligent Transportation Systems. 2016 Ieee 19th International Conference on Intelligent Transportation Systems (Itsc), 2663-2668.

37. Yue, X., Wang, H. J., Jin, D. W., Li, M. Q., \& Jiang, W. (2016). Healthcare Data Gateways: Found Healthcare Intelligence on Blockchain with Novel Privacy Risk Control. Journal of Medical Systems, 40(10), 8

38. Zhang, Y., \& Wen, J. T. (2015). An IoT Electric Business Model Based on the Protocol of Bitcoin. In 2015 8th International Conference on Intelligence in Next Generation Networks (pp. 184-191). New York: Ieee.

39. Zyskind, G., Nathan, O., \& Pentland, A. (2015). Decentralizing Privacy: Using Blockchain to Protect Personal Data. 2015 Ieee Security and Privacy Workshops (Spw), 180-184. 